\title{
Centroamérica Globalización, Tratado de Libre Comercio con los Estados Unidos y Metropolización Transnacional*
}

\author{
Mario Lungo
}

Resumo: Entre outros processos vinculados ao processo de globalização, dois se destacam nesse trabalho: um sobre o Tratado de Livre Comércio entre os Estados Unidos e a América Central; o outro sobre a tendência para a metropolização de caráter transnacional nos países da região, que possuem antecedentes na reduzida vinculação histórica e econômica destes países. 0 Mercado Comum da América Central nos anos 60 , se soma com a criação do Sistema de Integração Centroamericana dos anos 90, e recentemente o Tratado de Livre comércio com os Estados Unidos, perante o qual se estabelece a hipótese preliminar de que estamos diante de uma tendência de Metropolização Transnacional.

Palavras-chave: Globalização, Integração Regional, Livre Comércio, Metropolização Transnacional

Resumen: Entre otros procesos vinculados al proceso de globalización, dos se destacan en este trabajo: uno, el Tratado de Libre Comercio entre los Estados Unidos y Centroamérica; y dos, una tendencia hacia una metropolización de carácter transnacional en los países de la región, que tiene antecedentes en la estrecha vinculación histórica y económica de estos países. Al Mercado Común Centroamericano en los años 60, se suma la creación del Sistema de Integración Centroamericana en los años 90, y recientemente el Tratado de Libre Comercio con los Estados Unidos, ante lo cual se plantea la hipótesis preliminar de que estamos en presencia de una tendencia de Metropolización Transnacional.

Palabras-clave: Globalización, Integración Regional, Libre Comercio, Metropolización Transnacional

\section{Introducción}

En los países centroamericanos, como en el resto del continente, el proceso de metropolización en los años iniciales del siglo XXI es una tendencia ineludible. En los países de esta región presenta, no obstante, un rasgo particular, derivado de su reducida dimensión territorial, el alto grado de primacía urbana existente y la profunda vincula-

Presentado a la VIII Reunión de la Red Iberoamericana de Investigadores sobre Globalización y Territorio/ Grupo Globalización y Expansión Metropolitana/ Rio de Janeiro / Mayo 2004.

** Profesor y Investigador del Departamento de Organización del Espacio, Universidad Centroamericana "José Simeón Cañas", San Salvador.E-mail: lungo@integra.com.sv, jefadoe@buho.uca.edu.sv 
ción entre sus economías. Las primeras nos han llevado a sostener que estamos frente ante al desarrollo de ciudades grandes en países pequeños (Lungo, 2000). La última se está modificando debido al proceso de globalización y al Tratado de Libre Comercio Estados Unidos / Centroamérica acordado a finales del 2003.

El rasgo que particulariza a este proceso puede ser planteado en la forma de la hipótesis siguiente:

En Centroamérica tiende a conformarse una red urbana transnacional basada en el desarrollo de las principales áreas metropolitanas de cada país, subordinando a las redes urbanas nacionales. Este proceso de metropolización se verá acentuado por la implementación del Tratado de Libre Comercio Estados Unidos / Centroamérica y la integración de los países de la región.

De conformarse esta red urbana centroamericana, este hecho tendría profundas implicaciones para el desarrollo territorial y socio-económico de los distintos países, y para la gestión y la gobernabilidad urbanas en los mismos, modificando el panorama de la región al irse creando las posibilidades de un desarrollo territorial que desborde las fronteras nacionales en el marco de un creciente y contradictorio proceso de integración.

La afirmación tiene resonancias de procesos que se viven actualmente en Europa (Comisión Europea, 2001), y en zonas fronterizas como la existente entre México y los Estados Unidos, entre otros casos. Los requerimientos de la globalización y las desigualdades nacionales y regionales subyacen en estos procesos. Los países centroamericanos, con un nivel de desarrollo menor pero profundas vinculaciones históricas pudieran estar enfrentándose a un proceso de metropolización con elementos transnacionales que es necesario analizar para ver si la hipótesis planteada tiene fundamentos válidos.

Este trabajo constituye una aproximación primera a este planteamiento. No tiene, entonces, el objetivo de comprobar o rechazar la hipótesis anterior, lo que exigiría un trabajo de investigación. Pretende, únicamente, aportar algunos elementos e ideas para avanzar en su discusión y formulación, si ello es pertinente.

Se basa en investigaciones sobre el fenómeno urbano realizados en los años anteriores y en trabajos recientes sobre la migración internacional y sus impactos territoriales, fenómeno que está marcando el desarrollo de los países centroamericanos particularmente a partir de los años 80 .

\section{La evolución de las economías centroamericanas y de las principales áreas metropolitanas al iniciarse el siglo XXI}

El análisis de la evolución reciente de las principales áreas metropolitanas de Centroamérica requiere examinar, así sea brevemente, el desarrollo económico y social reciente en estos países. 


\section{El desarrollo actual de los países centroamericanos}

El Segundo Informe sobre Desarrollo Humano en Centroamérica y Panamá, recientemente presentado (PNUD, 2003), permite hacer una síntesis sobre las tendencias del desarrollo actual de los países centroamericanos ${ }^{1}$. De este Informe destacamos los procesos más importantes relacionados con el proceso de urbanización y la evolución de las principales áreas metropolitanas.

En general los países de la región han perdido el ritmo de crecimiento que tuvieron durante la primera mitad de los años 90 , a pesar de la estabilidad política, la apertura comercial y el nuevo impulso de la integración centroamericana. El débil crecimiento económico se ha concentrado en pocos sectores, excluyendo a las exportaciones tradicionales, la pequeña y mediana empresa y el sector agropecuario, los principales generadores de empleo.

Es visible la caída de la participación del sector agropecuario en los años 90 en el PIB nacional de El Salvador y Honduras; su estancamiento en Costa Rica y Guatemala; y el crecimiento en Nicaragua. Estas tendencias expresan, en general, la crisis en este sector y es una de las fuentes más importantes de expulsión de población fuera de los territorios nacionales, de la que no se excluye Nicaragua a pesar del incremento del PIB en este sector. Este proceso migratorio internacional y el crecimiento de la urbanización constituyen dos de los principales rasgos demográficos.

\section{Cuadro 1}

PIB agropecuario de los paises centroamericanos

\begin{tabular}{|l|c|c|c|c|}
\hline \multicolumn{1}{|c|}{ País } & $\mathbf{1 9 9 0}$ & $\mathbf{1 9 9 5}$ & $\mathbf{2 0 0 0}$ & $\mathbf{2 0 0 1}$ \\
\hline Costa Rica & 12.7 & 12.5 & 11.6 & 11.6 \\
\hline El Salvador & 16.5 & 13.4 & 12.1 & 11.6 \\
\hline Guatemala & 22.0 & 21.4 & 20.2 & 20.0 \\
\hline Honduras & 25.9 & 20.6 & 19.2 & 18.7 \\
\hline Nicaragua & 30.9 & 33.3 & 36.7 & 36.8 \\
\hline
\end{tabular}

Fuente: CEPAL, 2002, Panorama Social de América Latina.

\footnotetext{
${ }^{1}$ Aunque el Informe del PNUD incluye a Panamá, no hemos incorporado en este análisis a este país al no formar parte del la negociación del Tratado de Libre Comercio entre los Estados Unidos y Centroamérica. Por otra parte, sus peculiaridades históricas y económicas otorgan a este país características que lo diferencian sustancialmente, política y económicamente de los otros de la región.
} 
Aunque la pobreza relativa ha disminuido hoy hay más pobres que hace diez años y se ha incrementado la desigualdad social. La reducción ha sido más significativa en las áreas urbanas que en las rurales, mientras los países de la región experimentan un acelerado proceso de urbanización, estimándose para el año 2000 un 49.8\% de la población residía en áreas urbanas (PNUD, 2003).

Cuadro 2

Pobreza en Centroamérica

\begin{tabular}{|c|c|c|c|c|}
\hline \multirow{2}{*}{ País } & \multicolumn{4}{|c|}{ Población en situación de pobreza } \\
\cline { 2 - 5 } & Año & Medio & Medio & Total \\
& & Urbano & Rural & \\
\hline \multirow{2}{*}{ Costa Rica } & 1992 & 25.0 & 52.0 & 25.0 \\
& 2000 & 16.7 & 22.0 & 18.9 \\
\hline \multirow{2}{*}{ El Salvador } & 1992 & 43.1 & 55.7 & 48.3 \\
& 2000 & 36.7 & 53.7 & 43.8 \\
\hline \multirow{2}{*}{ Guatemala } & 1986 & 54.0 & 75.0 & 68.0 \\
& 2000 & 39.8 & 79.9 & 64.4 \\
& 1992 & 56.0 & 46.0 & 50.0 \\
Honduras & 2000 & 63.7 & 70.3 & 67.3 \\
\hline \multirow{2}{*}{ Nicaragua } & 1993 & 31.9 & 76.1 & 50.3 \\
& 2000 & 44.9 & 75.7 & 58.9 \\
\hline
\end{tabular}

Fuentes: CEPAL, Panorama Social de América Latina; Banco Mundial, World Development Indicators y PNUD Human Development Report (varios años)

La desigualdad en el crecimiento también se manifiesta al nivel sectorial en cada país y entre éstos. Mientras se ha avanzado en lo que se refiere a las políticas macroeconómicas y la dotación de infraestructura, se observan importantes retrocesos en las políticas de transformación productiva y la vulnerabilidad comercial. Esta última muestra una alta concentración de las exportaciones hacia los Estados Unidos.

El Informe sobre Desarrollo Humano para Centroamérica no aborda en detalle, paradójicamente, el análisis del proceso de urbanización, limitándose a constatar que la metropolización desordenada incrementa la vulnerabilidad de la región y que el XXI será un siglo urbano en Centroamérica. El Informe estima que la población urbana, actualmente el $50 \%$ de la población total se duplicará en aproximadamente veinte años. Agrega que en el año 2000 cuatro países centroamericanos se encontraban entre las veinte y cinco naciones del mundo con los niveles más altos de primacía urbana, desta- 
cándose Guatemala con 71.8\% de la población urbana total, San José de Costa Rica con el 51.3\% y el Área Metropolitana de San Salvador con 48.1\%.

El Informe destaca las diferencias entre los distintos países de la región, los que tienen una explicación histórica y vienen desde la época colonial, continuaron luego de la independencia de España en 1821, y se acentuaron a lo largo del siglo XX. En este momento de apertura estas diferencias son evidentes en los indicadores utilizados para medir el grado de preparación para participar en Tratados de Libre Comercio y la competitividad de los distintos países².

Cuadro 3

Centroamérica: grado de preparación para participar

en el ALCA. 1999, 2001

\begin{tabular}{|c|c|c|c|c|c|c|c|c|c|c|}
\hline \multirow{2}{*}{ Indicadores } & \multicolumn{2}{|c|}{ Costa Rica } & \multicolumn{2}{|c|}{$\begin{array}{c}\text { EI } \\
\text { Salvador }\end{array}$} & \multicolumn{2}{|c|}{ Guatemala } & \multicolumn{2}{|c|}{ Honduras } & \multicolumn{2}{|c|}{ Nicaragua } \\
\hline & 1999 & 2001 & 1999 & 2001 & 1999 & 2001 & 1999 & 2001 & 1999 & 2001 \\
\hline $\begin{array}{l}\text { Indicadores de } \\
\text { Elegibilidad }\end{array}$ & 48 & 72 & 72 & 88 & 68 & 64 & 52 & 64 & 28 & 52 \\
\hline $\begin{array}{c}\text { Estructura } \\
\text { macroeconómica } \\
\text { y sectorial }\end{array}$ & 43 & 37 & 43 & 50 & 37 & 30 & 43 & 40 & 50 & 50 \\
\hline $\begin{array}{c}\text { Inserción } \\
\text { internacional }\end{array}$ & 53 & 73 & 67 & 73 & 47 & 53 & 47 & 47 & 53 & 60 \\
\hline $\begin{array}{l}\text { Recursos } \\
\text { humanos }\end{array}$ & 80 & 90 & 50 & 60 & 50 & 30 & 35 & 40 & 40 & 30 \\
\hline Infraestructura & 80 & 93 & 20 & 60 & 20 & 40 & 20 & 40 & 40 & 27 \\
\hline $\begin{array}{c}\text { Politica } \\
\text { macroeconómica }\end{array}$ & 43 & 33 & 50 & 53 & 60 & 60 & 30 & 50 & 33 & 52 \\
\hline $\begin{array}{l}\text { Politica } \\
\text { comercial }\end{array}$ & 80 & 80 & 73 & 80 & 73 & 80 & 73 & 80 & 80 & 70 \\
\hline $\begin{array}{l}\text { Politica de } \\
\text { transformacion } \\
\text { productiva }\end{array}$ & 60 & 65 & 40 & 35 & 40 & 40 & 45 & 45 & 45 & 35 \\
\hline $\begin{array}{l}\text { Vulnerabilidad } \\
\text { comercial }\end{array}$ & 47 & 40 & 53 & 20 & 60 & 33 & 40 & 27 & 27 & 20 \\
\hline $\begin{array}{l}\text { Vulnerabilidad } \\
\text { macroeconómica }\end{array}$ & 64 & 72 & 32 & 56 & 44 & 60 & 52 & 52 & 36 & 36 \\
\hline
\end{tabular}

Fuente: CEPAL, 2003

Pero es también el creciente debilitamiento institucional interno uno de los rasgos sobresalientes del desarrollo centroamericano en la pasada década, a pesar del avance de los procesos de democratización política y de los intentos de integración de los países del

\footnotetext{
Es necesario criticar los criterios para medir la competitividad del Global Economic Forum. En el caso centroamericano se ha hecho un interesante estudio que identifica cuatro clusters potenciales para una inserción más competitiva de la región en la globalización: turismo, industria textil, agroindustria de alto valor agregado e industrias de componentes electrónicos y producción de software (INCAE \& HIID, 1999). Sin embargo la migración internacional y sus efectos no están incorporados en el análisis. Sería interesante revisar estas propuestas a las luz del Tratado de Libre Comercio y las remesas enviadas por los migrantes.
} 
istmo. Esta situación contrasta con los acuerdos tomados en las frecuentes reuniones de los presidentes centroamericanos.

Desde 1991 se han realizado 31 reuniones cumbres donde se han adoptado 30 instrumentos jurídicos y se han emitido 68 declaraciones, sobre una diversidad muy amplia de temas. Sin embargo, la cuestión específica de la urbanización y la metropolización no está bajo la responsabilidad directa de ninguna de las secretarias e instituciones especializadas del Sistema de Integración Centroamericano (SICA), creado en 1991.

Así, el desarrollo reciente de los países centroamericanos presenta un panorama complejo de cara a la globalización y la firma de Tratado de Libre Comercio con los Estados Unidos, mientras es previsible que la migración hacia los Estados Unidos y Canadá continúe al mismo ritmo actual sino se incrementa por los impactos del tratado en el sector rural de la región, incidiendo en los procesos de urbanización y metropolización, lo que por el contrario tenderán a verse potenciados con la operación de este Tratado.

\section{La globalización en Centroamérica y su espacialidad}

Las redes urbanas de los distintos países del istmo se caracterizan por la continuidad de la primacía urbana prevaleciente desde inicios del siglo XX y la configuración de áreasregiones metropolitanas cuyos vínculos principales comienzan a establecerse con otras ciudades del mundo. Lo anterior no debe conducir a hablar prematuramente de ciudades o regiones globales en Centroamérica, pero es indudable que hay indicios de que las principales ciudades se han configurado como áreas o regiones metropolitanas, tendiendo a potenciar las vinculaciones transnacionales aumentando las diferencias y tensiones con sus territorios nacionales.

Además de los rasgos comunes del proceso de globalización en todo el mundo, en Centroamérica es posible identificar manifestaciones específicas en la región, derivadas de su historia común y la estrecha interrelación existente entre los países del istmo. Entre las más importantes de estas manifestaciones de ésta última se encuentran:

a. En el aspecto económico, la globalización tiende a dar un nuevo impulso al proceso de integración que, iniciado en los años 60, se había estancado profundamente. Esto guarda relación con el reducido tamaño de las economías de estos países, que aisladamente les impide una inserción competitiva en el nuevo contexto mundial.

b. En la dimensión social, la conformación tanto de una nueva élite dominante como de amplios sectores populares impregnados de valores altamente transnacionales, basados, los de los primeros, en la profundización de las relaciones comerciales internacionales, y en los segundos en los intensos flujos migratorios internacionales. 
c. De aquí se desprenden importantes consecuencias políticas: el debilitamiento de las concepciones de desarrollo limitadas por las fronteras nacionales en diferentes sectores de la población con vínculos transnacionales, que tienen la mayor cuota de poder económico y político en casi todos los países centroamericanos y caribeños, y que se contraponen a las opciones de los sectores menos integrados al proceso de globalización (los agricultores y los industriales cuyas actividades se orientan a los mercados internos entre ellos), que conforman los sectores con menor poder de decisión económica y política.

En términos espaciales, el proceso de globalización en Centroamérica se caracteriza, además de la coexistencia de ciudades-regiones integradas a los circuitos internacionales de la economía y desintegradas en buena medida del resto de sus territorios nacionales, por la incipiente configuración de un sistema de ciudades-regiones metropolitanas articuladas espacialmente al nivel de la región centroamericana, tal como lo sugerimos en la hipótesis mencionada al inicio.

Lo anterior no es una tendencia nueva. Encontramos inicios de la configuración de un sistema de ciudades centroamericanas en los años 60, a raíz de la creación del Mercado Común Centroamericano bajo el impulso de las políticas desarrollistas y de instituciones como la CEPAL. La diferencia con los años actuales es que este proceso entra en contradicción con las tendencias de descentralización institucional y económica (que avanza lentamente en la región), y los planteamientos ambientalistas al generar contradicciones entre la configuración de esta red urbana metropolitana de carácter regional y el Corredor Biológico Mesoamericano, base de la política de desarrollo sostenible del istmo hasta el momento actual. Pero por otra parte este proceso recibe un fuerte impulso con el Plan Puebla-Panamá que propone la construcción de un Corredor Logístico Centroamericano y el recientemente acordado Tratado de Libre Comercio Estados Unidos / Centroamérica.

\section{Las economías metropolitanas en Centroamérica}

Las economías de las principales ciudades centroamericanas al iniciarse el siglo XXI se caracterizan por un claro predominio de las actividades comerciales y de servicios, unas orientadas a los mercados internos y centroamericanas, otras, cada vez más importantes, vinculadas a ciudades fuera del istmo en un creciente proceso de transnacionalización que va desdibujando las fronteras centroamericanas.

Estas contradicciones pueden ser la fuente de conflictos al interior de cada país al acentuar las disparidades regionales y provocar una segmentación espacial en las ciudades al crearse islotes urbanos modernos, y entre los distintos países del istmo al profundizarse la competencia entre las principales ciudades por atraer las inversiones extranjeras y ser la sede de los centros de decisión económica y política de carácter transnacional. 
Adicionalmente, al partir de una situación de gran desigualdad social y economías urbanas altamente informalizadas, el desarrollo de las ciudades más dinámicas tenderá a aumentar la segregación socio-espacial existente y incrementar la generación de riesgos urbanos. Las discusiones en algunas ciudades sobre grandes proyectos de infraestructura presagian lo que puede suceder. El caso del proyectado periférico para el Área Metropolitana de San Salvador o la reubicación de los aeropuertos de Tegucigalpa o Guatemala son ejemplos al respecto.

Lamentablemente no existen análisis sobre las economías urbanas centroamericanas, contándose únicamente con un estudio específico para el caso del Área Metropolitana de San Salvador (Cuervo, 2002), y los resultados generales de una investigación reciente sobre la ciudad de Guatemala (Avancso, 2004). Se ha realizado otro estudio empírico que compara la situación del Área Metropolitana de San Salvador, Tegucigalpa y la ciudad de Panamá (Banco Mundial, 2002), pero se limita al análisis de los sectores urbanos pobres y el acceso al trabajo y los servicios urbanos, mostrando que la violencia, la insuficiencia en el suministro de agua potable, la deficiente recolección de desechos sólidos y el acceso vehicular constituyen los mayores problemas de los barrios pobres en las dos primeras ciudades. Estos resultados muestran la existencia de difíciles condiciones para la competitividad de estas ciudades.

\section{Cuadro 4}

Hogares pobres en el Area Metropolitana de

San Salvador y Tegucigalpa

\begin{tabular}{|l|c|c|}
\hline \multicolumn{1}{|c|}{ Caracteristicas } & $\begin{array}{c}\text { AMSS } \\
\text { (2000) }\end{array}$ & $\begin{array}{c}\text { Tegucigalpa } \\
\text { (2000) }\end{array}$ \\
\hline \% de hogares formados fuera del AMSS & 21 & 13 \\
\hline $\begin{array}{l}\text { \% de hogares que escogieron su barrio por } \\
\text { disponibilidad de suelo }\end{array}$ & 46 & 54 \\
\hline \% de cabezas de hogar sin ninguna educación & 67 & 64 \\
\hline $\begin{array}{l}\text { \% de cabezas de hogar que participan en le } \\
\text { mercado laboral }\end{array}$ & 22 & 17 \\
\hline$\%$ de cabezas de hogar desempleadas & 37 & 25 \\
\hline
\end{tabular}

Fuente: Banco Mundial (2002). 
También se realizó una investigación para examinar las condiciones de competitividad de las Áreas Metropolitanas de San Salvador y San José de Costa Rica, estudiando tres aspectos que inciden en la localización de nuevas empresas en estas ciudades: el transporte de personal y de bienes y servicios; la seguridad; la dotación de servicios urbanos básicos; y los costos de los trámites exigidos por las normas urbanísticas y ambientales (Freire y Polése, 2003), encontrándose las fuertes debilidades que presentan ambas ciudades en estos aspectos pero también que los principales factores que determinan la localización de los establecimientos en ambas ciudades son la presencia de los clientes, de empresas proveedoras y de firmas complementarias.

Cuadro 5

Comunicaciones. Conectividad en los países centroamericanos

\begin{tabular}{|l|c|c|c|c|}
\hline País & $\begin{array}{c}\text { Líneas } \\
\text { telefónicas } \\
\text { fijas por 100 } \\
\text { habitantes }\end{array}$ & $\begin{array}{c}\text { Suscrpciones } \\
\text { a celulares } \\
\text { por } 100 \\
\text { habitantes }\end{array}$ & $\begin{array}{c}\text { Computadoras } \\
\text { personales } \\
\text { por 100 } \\
\text { habitantes }\end{array}$ & $\begin{array}{c}\text { Usuarios } \\
\text { de internet } \\
\text { por 10,000 } \\
\text { habitantes }\end{array}$ \\
\hline Guatemala & 6.5 & 9.7 & 1.3 & 171.1 \\
\hline Costa Rica & 23.0 & 7.6 & 17.0 & 933.6 \\
\hline El Salvador & 9.3 & 12.5 & 2.2 & 79.7 \\
\hline Honduras & 4.7 & 3.6 & 1.2 & 61.7 \\
\hline Nicaragua & 3.1 & 3.0 & 1.0 & 98.7 \\
\hline
\end{tabular}

Fuente: International Telecommunication Union, 2002.

Estas condiciones se inscriben en un desarrollo de las telecomunicaciones también desigual entre los distintos países de la región.

Es difícil tener un panorama completo de la evolución de las economías de las principales metrópolis centroamericanas dado que no hay estudios específicos y comprensivos sobre éstas. Se ha elaborado, sin embargo, un sugestivo trabajo sobre la posición de las principales ciudades de la región en el espacio de los flujos globales (Brown, Catalano y Taylor, 2001), en la que se afirma que las principales ciudades de Centroamérica, a pesar de estar al margen de las ciudades mundiales, funcionan a través de éstas, configurando lo que llaman estos autores la operación de firmas mundiales en ciudades locales. En el trabajo se mide la conectividad de las cinco ciudades capitales de Centroamérica cuyos resultados aparecen en los cuadros siguientes. 


\section{Cuadro 6}

Representación por sectores de firmas mundiales en las capitales centroamericanas

\begin{tabular}{|l|c|c|c|c|c|c|c|}
\hline \multicolumn{1}{|c|}{ Ciudad } & AC & AD & BF & IN & LW & MC & Total \\
\hline San José & 10 & 5 & 3 & 1 & 0 & 1 & 20 \\
\hline Guatemala & 9 & 8 & 1 & 1 & 0 & 0 & 19 \\
\hline San Salvador & 8 & 5 & 3 & 0 & 0 & 1 & 17 \\
\hline Tegucigalpa & 6 & 4 & 1 & 0 & 0 & 1 & 12 \\
\hline Managua & 4 & 5 & 0 & 1 & 0 & 1 & 11 \\
\hline
\end{tabular}

$A C=$ contabilidad; $A D=$ publicidad $; B F=$ banca/finanzas, $I N=$ seguros; LW = leyes; $M C=$ asesoria administrativa

\section{Cuadro 7}

Conectividad mundial de las capitales centroamericanas ${ }^{3}$

\begin{tabular}{|l|c|c|c|}
\hline \multicolumn{1}{|c|}{ Ciudad } & $\begin{array}{c}\text { Posición } \\
\text { mundial }\end{array}$ & $\begin{array}{c}\text { Conectividad } \\
\text { absoluta }\end{array}$ & $\begin{array}{c}\text { Conectividad } \\
\text { relativa }\end{array}$ \\
\hline Guatemala & 130 & 11,470 & 0.18 \\
\hline San José & 139 & 11,076 & 0.18 \\
\hline San Salvador & 154 & 10,471 & 0.17 \\
\hline Tegucigalpa & 194 & 8,240 & 0.13 \\
\hline Managua & 226 & 6,334 & 0.10 \\
\hline
\end{tabular}

El análisis anterior muestra las diferencias existentes entre las tres principales ciudades: Guatemala, San José y San Salvador, las que se complementarían con la incorporación de las sedes de los principales bancos, empresas de transporte aéreo (San Salvador), sedes de los organismos de integración regional (San Salvador, Guatemala y Tegucigalpa), y de la empresas transnacionales de alta tecnología como INTEL (san José). Pero el

\footnotetext{
${ }^{3}$ Medición hecha basada en el procedimiento propuesto en el artículo Taylor P.J., Catalano G. Y Walker D. "Specification of the world city network", Ga WC Research Bulletin 43.
} 
análisis brinda pistas para apoyar lo planteado en la hipótesis inicial sobre el proceso de conformación de una red urbana centroamericana de carácter metropolitano. A las cinco ciudades capitales habría que agregar la segunda ciudad de Honduras, San Pedro Sula, principal polo económico urbano de ese país.

\section{El Tratado de Libre Comercio Estados / Centroamérica y otros instrumentos de integración regional}

Antes de analizar el Tratado de Libre Comercio Estados Unidos / Centroamérica y su relación con la metropolización, examinemos otros instrumentos que promueven la integración regional.

\section{EI Sistema de Integración Centroamericano (SICA)}

Centroamérica cuenta con un largo historial de integración regional cuya mayor expresión es el SICA, y que reúne a varias de las instituciones creadas en las décadas precedentes para impulsar la integración de los países de la región y cuya sede está en San Salvador. Como antecedentes mas destacados se encuentran la creación del Parlamento Centroamericano (Parlacen), en 1986; la creación del propio SICA en 1991, que incorpora a la Secretaría de Integración Económica Centroamericana (SIECA), fundada en 1960; la suscripción de la Alianza Centroamericana para el Desarrollo Sostenible (Alides), en 1994; y numerosos tratados y acuerdos sobre aspectos específicos.

Como decíamos antes, temas como la urbanización y la metropolización están ausentes de su agenda de trabajo, e incluso su papel ha sido marginal en la discusión del Tratado de Libre Comercio Estados Unidos / Centroamérica. Incluso un tema muy vinculado como esta problemática, como son las migraciones internacionales, no ocupa una posición central en su agenda.

El SICA aborda, a través del trabajo de sus secretarías técnicas e instituciones regionales, cuestiones como y las redes de infraestructuras y servicios. Sin embargo la mayoría de ellas reproduce la orientación sectorial de los gobiernos centroamericanos (economía, educación, salud, etc.), no existiendo instituciones para abordar temas transversales. El único ejemplo lo constituye la prevención de desastres naturales, para lo que se creó el Centro de Coordinación para la Prevención de Desastres Naturales en América Central (CEPREDENAC).

Respecto a otras cuestiones territoriales cuenta con unidades técnicas para los programas de vivienda y asentamientos humanos, transporte y los recursos hídricos. Existe, por otra parte, desde hace 39 años, el Banco Centroamericano de Integración Económica (BCIE), que ha cumplido un importante papel en el financiamiento de infraestructura urbana. 


\section{El Plan Puebla-Panamá}

Mayor incidencia en la temática que nos ocupa tiene el Plan Puebla-Panamá. A partir de una iniciativa del gobierno mexicano los Presidentes de la región acordaron impulsar en 1999 el Plan Puebla Panamá, que reúne una serie de iniciativas y políticas con el objetivo de desarrollar la región mesoamericana. Las políticas derivadas de este Plan se sumarán a las contenidas en el Tratado de Libre Comercio e influirán sin duda en las dinámicas urbana y metropolitana actuales.

El Plan Puebla Panamá plantea una estrategia integral para la región que ampara un conjunto de ocho iniciativas:

- desarrollo sustentable

- desarrollo humano

- prevención y mitigación de desastres naturales

- turismo

- facilitación del intercambio comercial

- interconexión vial

- interconexión eléctrica

- telecomunicaciones

Es evidente que el énfasis está siendo colocado en las cuatro últimas iniciativas, por lo que se comienzan a generar contradicciones entre el Corredor Biológico Mesoamericano y el Corredor Logístico Puebla-Panamáy que se relación indirectamente con el tema de este trabajo.

El objetivo del Plan Puebla-Panamá, según sus formuladores, es potenciar la riqueza humana y ecológica de la región, dentro de un marco de desarrollo sustentable que respete la diversidad cultural y étnica. Sin embargo, al analizar las iniciativas propuestas, se encuentra que el Plan prioriza la construcción de la infraestructura necesaria para lograr una mejor conectividad entre los países de la región y su inserción en la globalización. Por esta razón las principales iniciativas de este Plan se han concentrado en proyectos de infraestructura vial y de interconexión eléctrica.

Entre los primeros se destaca la construcción de la Red Internacional de Carreteras Mesoamericanas, con un costo de más de 3,500 millones de dólares (PNUD, 2003). Este proyecto implica impactos potenciales en los procesos migratorios que afectarán la urbanización y la metropolización en los países de la región. En general, los proyectos del Plan Puebla-Panamá constituyen un requisito básico para poder implementar las políticas impulsadas por el Tratado de Libre Comercio Estados Unidos / Centroamérica. 


\section{El Tratado de Libre Comercio Estados Unidos / Centroamérica}

La firma de un Tratado de Libre Comercio entre los Estados Unidos y Centroamérica ha ocupado un primer plano en las discusiones políticas y académicas en los países de la región, suscitando fuertes debates. Como es sabido el centro de las negociaciones es la liberalización comercial y sus impactos, especialmente para el sector agropecuario e industrial.

El texto que se está negociando consta de 20 Capítulos, de los cuales el comercio de bienes y servicios, así como todas las regulaciones que se aplicarán al intercambio comercial se desarrollan en 18 de ellos; los otros dos corresponde a un Capítulo Ambiental y a un Capítulo Laboral. Este último es el único que desarrolla políticas relacionadas escuetamente con las migraciones internacionales, en donde las remesas constituye una cuestión fundamental para la estabilidad económica de la mayoría de los países del istmo ${ }^{4}$.

Es claro, dadas las desigualdades crecientes, sociales y entre países, que las consecuencias del Tratado de Libre Comercio sobre las migración internacional y las remesas, también serán desiguales. Por ejemplo, al interior de Centroamérica esta tendencia podría afectar en forma particular a los migrantes nicaragüenses residentes en Costa Rica, que suman 225,000 personas ${ }^{5}$, asentados muchos de ellos en el Área Metropolitana de San José, que conforman el grupo más pobre de este país.

Los objetivos de Estados Unidos para impulsar el Tratado serían tres: promover las exportaciones de ese país, apoyar las reformas económicas y la democratización en la región, y estimular avances en relación al propuesto Tratado de Libre Comercio con América Latina (ALCA).

No se encuentran en el Tratado entre Estados Unidos y Centroamérica aspectos relacionados directamente con los procesos de urbanización y metropolización. Sin embargo, al estar la cuestión de la agricultura y la modernización de la economía en el centro del debate, es previsible que su transformación implique un aumento de las migraciones hacia los principales centros urbanos, especialmente las capitales que constituyen las áreas metropolitanas.

\footnotetext{
${ }^{4}$ Un informe del BID estima que América Latina recibirá este año cerca de 40,000 millones de dólares en remesas familiares, cifra que superará en más del 50\% a la inversión directa, prevista en 24,000 millones. En el año 2002, El Salvador recibió 2,206 millones, colocándose en la cuarta posición entre los países latinoamericanos, detrás de México, Brasil, y Colombia, pero teniendo una población sensiblemente menor que los anteriores y una reducida extensión geográfica (20,000 Km.2). Guatemala recibió 1,698 millones, Honduras 770 y Nicaragua 759 (El Diario de Hoy, 28 octubre, 2003).

${ }^{5} 5.4 \%$ de la población costarricense actual, según datos oficiales, aunque otros cálculos estiman que los migrantes nicaragüenses son casi medio millón de personas.
} 
Por otra parte, Centroamérica en los últimos años ha firmado además Tratados de Libre Comercio con otros países: México, Chile, República Dominicana y Panamá. Al revisar estos Tratados observamos que los cuatro responden a lineamientos y tienen una estructura similares. Estos documentos responden así, a una plantilla derivada del Tratado de Libre Comercio en América del Norte y no presentan variaciones sustantivas en su contenido.

El análisis de los documentos muestran que dichos Tratados enfatizan en aspectos comerciales, desarrollando una serie de regulaciones encaminadas principalmente a la apertura de mercados. Sin embargo, los documentos no se limiten exclusivamente a acuerdos comerciales, sino que también se incluyen distintos capítulos que tienden a incidir en las políticas económicas y sociales de las naciones suscritas, trascendiendo así, lo estrictamente comercial. Así, encontramos los apartados de inversión, compras del sector público, energía y petroquímica, telecomunicaciones, servicios financieros, transporte aéreo y propiedad intelectual.

\section{Otras propuestas y acuerdos bi o multilaterales}

Una de las iniciativas más importantes para impulsar la integración centroamericana fue formulada en 1999 a través de un proyecto de la universidad de Harvard y el Intituto Centroamericano de Administración de Empresas (INCAE), institución de formación empresarial apoyada por la primera. Este proyecto, que tenía por objetivo promover la competitividad de los países de la región de forma integrada (INCAEHIID,1999), sugirió la creación de cuatro clusters: turismo, agroindustria de alto valor agregado, textiles y manufactura de piezas electrónicas y servicios informáticos.

Un análisis de esta propuesta, que ha sido asumida por los principales empresarios privados, muestra que su implementación requeriría una fuerte modernización institucional y un desarrollo tecnológico que, sin duda, reforzaría el papel de las aglomeraciones urbanas más importantes de Centroamérica. Lo anterior se complementa con las fuertes inversiones que, en las principales ciudades están efectuados los grupos inmobiliarios más poderosos, desbordando claramente las fronteras nacionales (centros comerciales, hoteles, conjuntos habitacionales de alto estándar, etc.).

Por otra parte, los países centroamericanos han firmado con la Unión Europea, varios acuerdos de cooperación para el desarrollo. Estos están poco o nada relacionados con la urbanización y orientadas principalmente hacia las zonas rurales más pobres y vulnerables, pero han estado incorporando sin embargo cada vez más la cuestión del ordenamiento territorial, incidiendo por esta vía en la problemática de las ciudades.

Respecto a acuerdos entre países centroamericanos, en la última década han surgido diferentes iniciativas referidas a las zonas fronterizas, las que están al margen de la influencia de las principales concentraciones urbanas. 


\section{Los impactos ocultos para el futuro de la metropolización en Centroamérica}

A partir de lo antes expuesto surge una interrogante: ¿̨dado la poca consideración de la problemática urbana urbana y la metropolización en las agendas de desarrollo de los países centroamericanos, y en los instrumentos de integración regional y en el Tratado de Libre Comercio Estados Unidos / Centroamérica, cuál es la relevancia de su relación?

Esta interrogante pone en cuestión la hipótesis planteada la inicio de este trabajo. Sin embargo, sostenemos que, aunque esta problemática haya sido dejada de lado por los dirigentes políticos se mantiene subyacente con fuerza y debe ser abordada. Ignorarla solo conducirá a enfrentar sus consecuencias una vez que muchos de sus impactos negativos estén consolidados y sea difícil revertirlos y a perder las oportunidades que ofrece para un desarrollo urbano más equitativo y sostenible el actual proceso de integración de la región.

Cuadro 8

Potencialidades e impactos negativos del proceso de integración en el desarrollo metropolitano

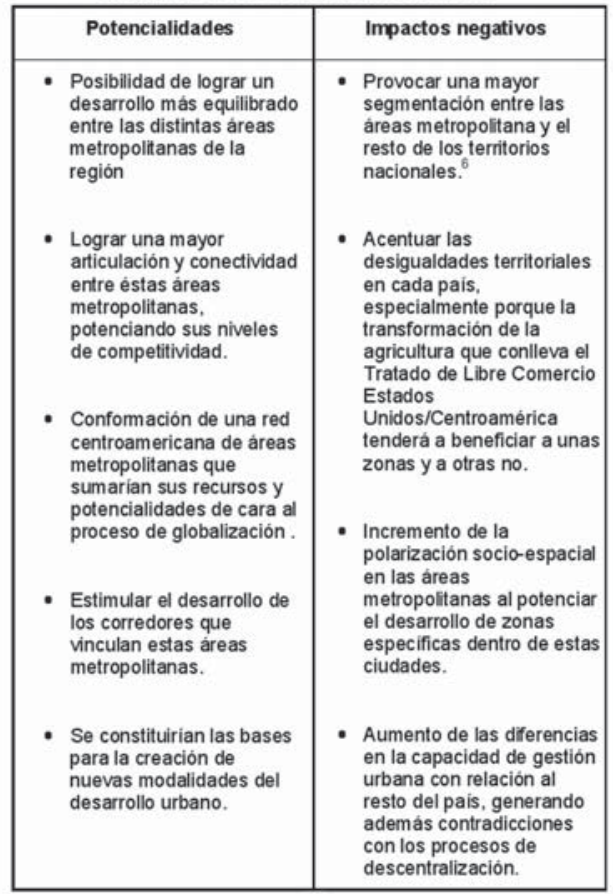

${ }^{6}$ Para reducir este impacto se deberán impulsar planes de ordenamiento territorial al nivel de cada país pero tomando en consideración las vinculaciones con los países vecinos (incluyendo México y Panamá). 
El desafío es identificar los impactos ocultos (algunos no lo son tanto pero no existe conciencia de ello), de la globalización y el proceso de integración regional en Centroamérica. Muchos de estos impactos, creemos, contribuirían a la conformación de la red urbana de carácter metropolitano en la región que hemos venido planteando. En el cuadro que se presenta a continuación se ha intentado esbozar las potencialidades y efectos negativos de la conformación en ciernes de una red metropolitana centroamericana.

Este esbozo de potencialidades e impactos negativos puede parecer una visión idealista influenciada por la planificación territorial tradicional que ignora las contradicciones sociales y el papel de los actores. Permite, no obstante, visualizar un escenario objetivo que no eluda las consecuencias de las tendencias crecientes a la metropolización en Centroamérica.

En estos años se han estado impulsando en varios de los países de la región planes de ordenamiento territorial y se esta tratando de promulgar leyes al respecto, los esfuerzos más avanzados son los de Honduras (donde se ha aprobado una Ley de Ordenamiento Territorial), y El Salvador, donde se ha formulado un Plan y un anteproyecto de ley. Aunque en el caso salvadoreño se recoge en el plan elaborado el innegable fenómeno metropolitano, expresado en el peso económico y político del Área Metropolitana de San Salvador con relación al resto del país, y se plantea que deberá jugar un papel al nivel centroamericano (VMVDU, 2004), hace falta realizar investigaciones en profundidad para abordar esta temática.

Podemos, sin embargo, avanzar algunas ideas en torno a la gestión del desarrollo de estas aglomeraciones metropolitanas.

\section{Los desafíos para la gestión metropolitana}

Ante el panorama descrito someramente en las páginas anteriores, es posible identificar los principales desafíos que se presentan para un desarrollo metropolitano equitativo y sostenible en el marco de una creciente integración donde el Tratado de Libre Comercio Estados Unidos / Centroamérica jugará un papel clave. Estos desafíos son los siguientes:

a. Se debe ampliar la búsqueda de la competitividad de las ciudades metropolitanas incorporando al incremento de la productividad urbana, una mejora sustancial en las condiciones de vida de sus habitantes, la sostenibilidad ambiental y la construcción de relaciones de gobernabilidad de carácter democrático.

b. Aunque es necesario incrementar la competitividad de las ciudades con la visión amplia antes descrita, evitando crear una segmentación espacial insostenible, es decir, rstringiendo que se creen zonas urbanas de relativa prosperidad en medio de un territorio donde impere la pobreza. Una adecuada política de ordenamiento territorial al nivel de cada país aparece como una necesidad y un complemento indispensable. 
c. Es indispensable crear nuevas formas de gestión urbana para las ciudades metropolitanas sin crear una atomización pero respetando los niveles locales; también es necesario incorporar los nuevos procesos transnacionales respetando los valores de las culturas locales y nacionales.

d. La reducción de la pobreza y la exclusión social urbanas en las áreas metropolitanas no pueden ser acciones complementarias. Tienen que estar en el centro de los planes y programas de desarrollo. El estudio reciente del Banco Mundial sobre el Área Metropolitana de San Salvador, Tegucigalpa y Panamá, permite identificar algunos puntos de intervención claves.

Es indispensable entonces, en general, incorporar en la gestión del desarrollo metropolitano en cada caso particular la dimensión regional, al nivel nacional, y la dimensión regional al nivel centroamericano.

\section{Conclusiones}

Queremos concluir este trabajo intentando responder a dos interrogantes que surgen de la exploración hecha:

¿ Puede la gestión urbana actual ir más allá de tratar de incrementar la competitividad de la economía de las principales ciudades centroamericanas?

Más allá del discurso y las intenciones, tanto la planificación urbana normativa, como la planificación urbana estratégica, que se entremezclan y compiten en la mayoría de países, tienen como objetivo principal en este momento incrementar la productividad de las economías urbanas y la competitividad de las ciudades para adecuarlas a la apertura comercial y a la globalización.

Con lo anterior no queremos negar el importante esfuerzo que numerosos planificadores en distintos países realizan cotidianamente con el objetivo de planificar un desarrollo urbano más equitativo y sostenible, ni la necesidad de incrementar la productividad de la economía urbana y la competitividad de las ciudades para alcanzar este objetivo, pero debe trascenderse la sola dimensión económica, lo que no es fácil en la medida en que, en tanto parte de las políticas públicas, la planificación se rige por los enfoques que predominan en ellas, y que no es factible una planificación alternativa al margen de las mismas.

Las principales reformas impulsadas desde finales de los años 80: apertura comercial, reforma financiera, modernización tributaria, privatización y flexibilización laboral, condujeron a enfocar las prioridades de la planificación en incrementar las productividad de las economías urbanas. Muy distante, más cerca del discurso ideológico que de las prácticas reales, se encuentra la descentralización.

Como es conocido, estas reformas se han traducido en un debilitamiento del Estado y de las políticas públicas, entre ellas las urbanas. La institucionalidad encargada del desarrollo 
de las ciudades, de paliar la exclusión social y las desigualdades espaciales, básicas para aumentar su competitividad también pierden peso y poder, llegándose, en algunos casos y momentos a plantear la necesidad de la reducción de la planificación a la mínima expresión e incluso en planteamientos extremos a la privatización de la gestión urbana, precisamente cuando por la acentuación de las desigualdades urbanas las ciudades se vuelven más injustas.

No obstante también se ha sostenido que para aumentar la competitividad de las ciudades es indispensable mejorar la educación y las condiciones de vida dela población más desfavorecida. Alrededor de esta necesidad vuelve a emerger la cuestión del derecho a la ciudad.

Para analizar si la planificación urbana, en cualquiera de sus modalidades, puede ir más allá de tratar de incrementar la competitividad de las ciudades, retomemos el camino seguido hace algunas décadas (Harvey, 1973), para identificar los procesos que, actualmente están en la base de la desigualdad urbana. Indudablemente las formas de propiedad y el uso de la tierra urbana, estrechamente asociada a la generación y captura de plusvalías, no solo inmobiliarias, sigue siendo la clave de la desigualdad urbana. Pero hay, en nuestra opinión, otros dos procesos derivados del modelo actual que se traducen en obstáculos para que la planificación intente corregir esta desigualdad:

- primero, la desregulación y la privatización que están en la esencia de este modelo;

- segundo, las configuración de estructuras sociales urbanas cada vez más complejas que, sumadas a los profundos cambios culturales ocurridos en los últimos años, dificultan el diseño de políticas que luchan contra la desigualdad urbana, entre las cuales se destacan las estructuras sociales metropolitanas.

Si lo primero es relativamente claro y su manejo depende de las de las condiciones y capacidades políticas, lo segundo es difícil de comprender porque ha sido poco investigado y de manejar por su gran complejidad. Por ejemplo, las formas de segregación socioespacial actuales constituyen un denso conjunto de manifestaciones cuyas relaciones no son siempre explícitas (Marcuse and van Kempen, 2000).

Para que la planificación urbana y metropolitana vaya más allá de proponerse el incremento de la competitividad de las ciudades tendría que incidir en cuestiones como las anteriores, para lo cual no está pensada y que la colocaría a contracorriente de las políticas públicas actuales. Por eso es que aún planteamientos hechos a partir de experiencias de gestión urbana en todo el mundo, que incluyen temas como la pérdida de la cohesión social o el debilitamiento de la institucionalidad urbana, que paradójicamente al nivel global parecen ser semejantes en los países del mundo desarrollado y en desarrollo, pero que sus significados son locales y subjetivos por la complejidad de las estructuras sociales urbanas actuales (Cohen, 1996), son poco abordados por los planificadores. 
¿Es posible impulsar una gestión metropolitana que permita aprovechar el actual proceso de integración en Centroamérica?

Ante todo se debe pasar de la limitada concepción de planificación a la visión más amplia de gestión urbana y metropolitana, que incluya además de la planificación, la regulación del desarrollo urbano, la administración de las ciudades y el financiamiento necesario para implementar este desarrollo.

Luego tienen que crearse nuevos instrumentos de gestión metropolitana que se contrapongan a las tendencias de desregulación y privatización. Es claro que si la sociedad pierde la capacidad de regular los procesos del mercado las desigualdades urbanas no cesarán de crecer y los conflictos de agudizarse, pero de lo que se trata es de repensar la regulación urbana misma, ya que en la mayoría de sus expresiones actuales sólo contribuye a legitimar la segregación espacial y la exclusión social.

Y lo que es más difícil: la incorporación del análisis de las nuevas estructuras sociales metropolitanas para poder incidir en las desigualdades urbanas. Aquí consideramos que es útil trabajar con los conceptos de identidad y diferencia sociales, y su relación con el poder y espacialidad, aunque parezcan a primera vista cuestiones muy alejadas de las práctica cotidiana de la gestión de las ciudades.

Partimos de una constatación: las ciudades están desigualmente ubicadas en la geometría global del poder (Massey, 1997), a lo que agregamos que los distintos sectores sociales, al interior también acceden desigualmente al poder, que en esto la espacialidad, en términos de localización, acceso a los bienes y servicios urbanos, es un factor clave, y que esto inciden en la constitución de las identidades urbanas y en la visión y lucha por el derecho a la ciudad.

Pero la identidad y la diferencia no puede verse simplemente alrededor de comunidades específicas. La espacialidad implica, en las ciudades, una mayor complejidad. Por eso es necesario nuevas visiones como las propuestas para construir ciudades socialmente sostenibles (Polèse, 2000).

Para ello es necesario impulsar políticas urbanas incluyentes, que estimulen la convivencia social de distintos grupos, que integren espacialmente una ciudad ahora fragmentada, que posibiliten una mayor igualdad económica y una amplia y permanente participación política, para lo cual propone la construcción de "puentes urbanos" que vinculen lo institucional con lo territorial alrededor de áreas de acción concretas como la gobernabilidad; las políticas sociales y culturales; la infraestructura social y servicios públicos; la tierra urbana y vivienda; el transporte y accesibilidad; el empleo y la revitalización económica, los espacios públicos; etc.

Volvemos de nuevo a los temas cercanos a los planificadores, con los cuales han trabajado durante décadas, ¿cuál es lo nuevo entonces? Se trata de abordar el desarrollo metropolitano con una nueva perspectiva que, en el caso centroamericano, debe tomar 
en consideración el proceso de integración que preludia la conformación de una red urbana metropolitana de carácter regional.

\section{Bibliografia}

AVANCSO Elproceso de crecimiento metropolitano de la ciudad de Guatemala. Guatemala, 2003.

BANCO MUNDIAL. La situación de los pobres respecto a la prestación de servicios urbanos: el caso de tres ciudades centroamericanas, Washington, 2002.

BROWN, E.; CATALANO, G.; TAYLOR, J. P. Más allá de las ciudades mundiales: América Central en un espacio de flujos global, en Espacios \# 15, FLACSO, San José, 2001.

CEPAL Panorama social de América Latina. Santiago de Chile, 2002.

CEPAL Grado de preparación para participar en el ALCA. (inédito), México, 2003.

COHEN, Michael. The Hypothesis of Urban Convergence: Are Cities in the North and South Becoming More Alike in an Age of Globalization, in Urban future.global pressues and local forces, M.Cohen, B. Ruble, J. Tulchin and A. Garland (editors), Woodrow Wilson Center, Washington, 1996.

COMISIÓN EUROPEA. Politica regional europea, Bruselas, 2001.

CUERVO, Luis Mauricio. La economía del área metropolitana de San Salvador. San Salvador: Banco Mundial / Oficina de Planificación del Área Metropolitana de San Salvador (OPAMSS), 2002.

FREIRE, Mila and POLĖSE, Mario. Connecting cities with macroeconomic concerns: teh missing link. Washington: The World Bank, 2003.

HARVEY, David Social justice and the city. London: E. Arnold Publishers, 1973.

INCAE (Centro Latinoamericano para la competitividad y el desarrollo sostenible) \& HIID (Harvard Institute for International Development) Centroamérica en el siglo XXI. Una agenda para la competitividad y el desarrollo sostenible. Bases para una discusión sobre el futuro de la región. Costa Rica: Alajuela, 1999.

LUNGO, Mario "Ciudad grande, país pequeño. Los desafíos de la gestión metropolitana en Centroamérica”, en Repensando a experiência urbana da América Latina: questoes, conceitos e valores, Ana Clara Torres Ribeiro (organizadora). Buenos Aires: CLACSO, 2000.

LUNGO, Mario La migración internacional y el Tratado de Libre Comercio Estados Unidos / Centroamérica, ponencia presentada a la conferencia sobre Migración y Desarrollo. Tijuana: El Colegio de la Frontera Norte, noviembre, 2003.

MARCUSE, Peter and VAN KEMPEN, Ronald Globalizing Cities. A New Spatial Order? Oxford: Blackwell Publishers, 2000. 
MASSEY, Doreen. Space/Power, Identity/Difference: Tensions in the City. in: The urbanization of injustice, Andy Merrifield and Erik Swyngedouw (editors). New York: New York University Press, 1997.

PNUD Segundo Informe sobre desarrollo humano en Centroamérica y Panamá. San José, 2003.

POLÉSE, Mario. Learning from Each Other: Policy Choices and the Social Sustainability of Cities, In: The social sustainability of cities, edited by M. Polése and R. Stren. Toronto: University of Toronto Press, 2000.

SICA Memoria de labores 2001. San Salvador, 2002.

SICA. Institucionalidad regional. In: http:www.sgsica.org/instituciones/index.php, 2003. Viceministerio de Vivienda y Desarrollo Urbano Plan Nacional de Ordenamiento y DesarrolloTerritorial. San Salvador, 2004. 
Mario Lungo - Cadernos PROLAM/USP (ano 3 - vol. 2 - 2004), p. 07-27 